\title{
Absence of an Acinar Gradient for Bile Acid Uptake in Developing Rat Liver
}

\author{
FREDERICK J. SUCHY, WILLIAM F. BALISTRERI, JOANETTE S. BRESLIN, \\ RANJANA DUMASWALA, KENNETH D. R. SETCHELL, AND SANFORD A. GARFIELD \\ Department of Pediatrics and Anatomy and Cell Biology, University of Cincinnati College of Medicine, \\ Cincinnati, Ohio 45267
}

\begin{abstract}
We studied the acinar distribution for uptake of the bile acid analogue $\left[{ }^{125} \mathrm{I}\right]$-cholylglycyltyrosine in livers from adult and 14-day-old suckling rats. Portal and peripheral (systemic) serum bile acid concentrations were also measured by combined gas chromatography-mass spectrometry as an independent index of hepatic bile acid clearance from portal blood. Utilizing light microscopic autoradiography, a steep, decreasing portal to centrilobular gradient for cholylglycyltyrosine uptake was noted in adult rat liver. In contrast, there was no lobular gradient for cholylglycyltyrosine uptake visible in the 14-day-rat liver; all hepatocytes within the acinus contained a similar number of silver grains. Portal vein total bile acid concentrations were significantly higher in serum of adult compared to 14-day-old rats. In contrast, bile acid concentrations were 10 -fold higher in the peripheral serum of developing versus adult rats. The peripheral to portal serum bile acid concentration ratio was 0.23 in the adult and 6.48 in the 14-day-old rat. We conclude that the entire hepatic lobule participates in the uptake of bile acids in the 14-day-old rat even under the basal conditions of this study. The normal "reserve" function of centrilobular hepatocytes is not sufficient to compensate for the decreased transport capacity of the developing liver with the result that increased concentrations of bile acids enter and accumulate in the systemic circulation. (Pediatr Res 21: 417-421, 1987)
\end{abstract}

\section{Abbreviations}

CGT, cholylglycyltyrosine

BSP, dibromosulfophthalein

A primary cellular event in bile formation involves the saturable, $\mathrm{Na}^{+}$-dependent uptake of bile acids from portal blood at the basolateral (sinusoidal) membrane of the hepatocyte (1). In the mature liver, the uptake process is normally so efficient that $85-90 \%$ of the conjugated bile acids are removed from portal blood during a single pass through the sinusoids (2). Several studies have directly shown by light microscope autoradiography that there is a steep, decreasing portal to centrilobular gradient for bile acid uptake in adult rat liver $(3,4)$. Such an acinar distribution for uptake is possible only with solutes that are very avidly extracted from portal blood.

Received May 2, 1986; accepted December 2, 1986.

Correspondence Frederick J. Suchy, M.D., Division of Pediatric Gastroenterology and Nutrition, Children's Hospital Research Foundation, Elland and Bethesda Avenues, Cincinnati, $\mathrm{OH} 45229$.

Supported in parts by Grants HD-20632 and HL-0727-5,2-05800-1391 from the US Public Health Service and the March of Dimes Birth Defects Foundation.
There is considerable evidence that during development bile acid uptake by the liver is decreased in comparison with the adult (5). Impaired bile acid clearance by the liver is suggested by elevated concentrations of serum bile acids in normal human infants and in suckling rats $(6,7)$. Plasma disappearance and biliary excretion of labeled taurocholate occurs at a much slower rate in suckling compared to adult rats (8). Recent studies utilizing hepatocytes and basolateral liver plasma membrane vesicles prepared from developing rats have shown that bile acid uptake is markedly decreased probably as a result of fewer functional bile acid carriers on the sinusoidal membrane of the hepatocyte $(9,10)$. These findings led us to the hypothesis that the low transport capacity of the developing liver for bile acids might well result in an acinar distribution for bile acid uptake which differed from the adult. Therefore we utilized light microscope autoradiography to study the acinar distribution for uptake of the bile acid analogue, CGT, in livers from adult and 14-dayold rats following portal vein injection of the isotope. We also determined serum bile acid concentrations in peripheral and portal vein serum from both age groups as a measure of hepatic bile acid clearance.

\section{MATERIALS AND METHODS}

Animals. Adult male Sprague-Dawley rats, weighing 150-200 $\mathrm{g}$, and 14-day-old rats with their dams, obtained from the Charles River Breeding Laboratories (Wilmington, MA), were housed in a temperature-controlled room at $22^{\circ} \mathrm{C}$ with 12 -h light-dark cycles. Adult rats were maintained on Purina Rat Chow and water ad libitum; pups were suckled by their dams. Animals were studied between 0800 and $0900 \mathrm{~h}$ in the fed state.

Tracer. CGT was prepared as previously described (11) and was then iodinated by the chloramine-T method to a specific activity of $475 \mu \mathrm{Ci} / \mu \mathrm{g}$. Hepatic transport properties of this compound have previously been reported (11). In brief, uptake of CGT by isolated hepatocytes was $\mathrm{Na}^{+}$-dependent, rate constants for its uptake were similar to native conjugated bile acids, and the tracer could be quantitatively recovered intact in bile over a time course similar to that of $\left[{ }^{14} \mathrm{C}\right]$-cholylglycine (11). CGT is cross-linked to cellular protein during glutaraldehyde fixation and therefore may be utilized for autoradiography.

Light microscope autoradiography. Adult and suckling rats underwent laparotomy under pentobarbital sodium anesthesia (5 mg/kg intraperitoneally). [ $\left.{ }^{125} \mathrm{I}\right]-\mathrm{CGT}(40 \mu \mathrm{Ci} / 100$ body weight $)$ was injected into the portal vein with a 27 -gauge needle over 15 $s$ (three animals used at each age). Thirty $s$ after injection of the tracer a portion of the right medium lobe of the liver was quickly excised and fixed in $2 \%$ glutaraldehyde $-2 \%$ paraformaldehyde in $0.1 \mathrm{M}$ cacodylate buffer at $\mathrm{pH} 7.3$ (modified from Karnovsky) (12). After fixation, portions of tissue were subdivided into 1.0$\mathrm{mm}^{3}$ pieces and immersed in the same fixative for an additional $2 \mathrm{~h}$ at room temperature. Samples were rinsed with $0.1 \mathrm{M}$ 
cacodylate buffer (pH 7.3), postfixed in $1 \%$ cacodylate-buffered osmium tetroxide, dehydrated with graded ethanol solutions, and embedded in Epon 812 (13).

Light microscope autoradiography was performed using $1-\mathrm{mm}$ thick sections of Epon-embedded specimens affixed to glass slides and coated under an appropriate safelight with Kodak NTB-2 emulsion (11). After exposure (10 days) they were developed using a D-19 developer, stained using toluidine blue, and cover slipped for viewing using dark field and bright field light microscopy.

Only sections showing a clearly defined terminal portal triad (less than $40 \mu \mathrm{m}$ in diameter) and central vein ("classical lobule") were utilized for further study. A typical section from three adult and three suckling rat liver preparations was used for quantitation of grains according to a modification of the method of Jones et al. (3). A grid square overlay was placed over light photomicrographs so that the grid squares lined up between the limiting plate of the portal triad and the central vein wall. The lobule was subdivided into four quarters from portal to central regions and the number of grains within each quarter was counted by punching a hole in each grain on the photomicrograph with a pin.

Determination of serum bile acid concentrations in rat plasma. One $\mathrm{ml}$ of blood was obtained from a peripheral (systemic) venous site and $0.5 \mathrm{ml}$ from the portal vein of adult male rats. Similar volumes were obtained by pooling blood samples from 14-day littermates. However portal vein phlebotomy was limited to a volume of 50-100 $\mu \mathrm{l}$ from each suckling animal to minimize contamination of portal venous blood with systemic blood.
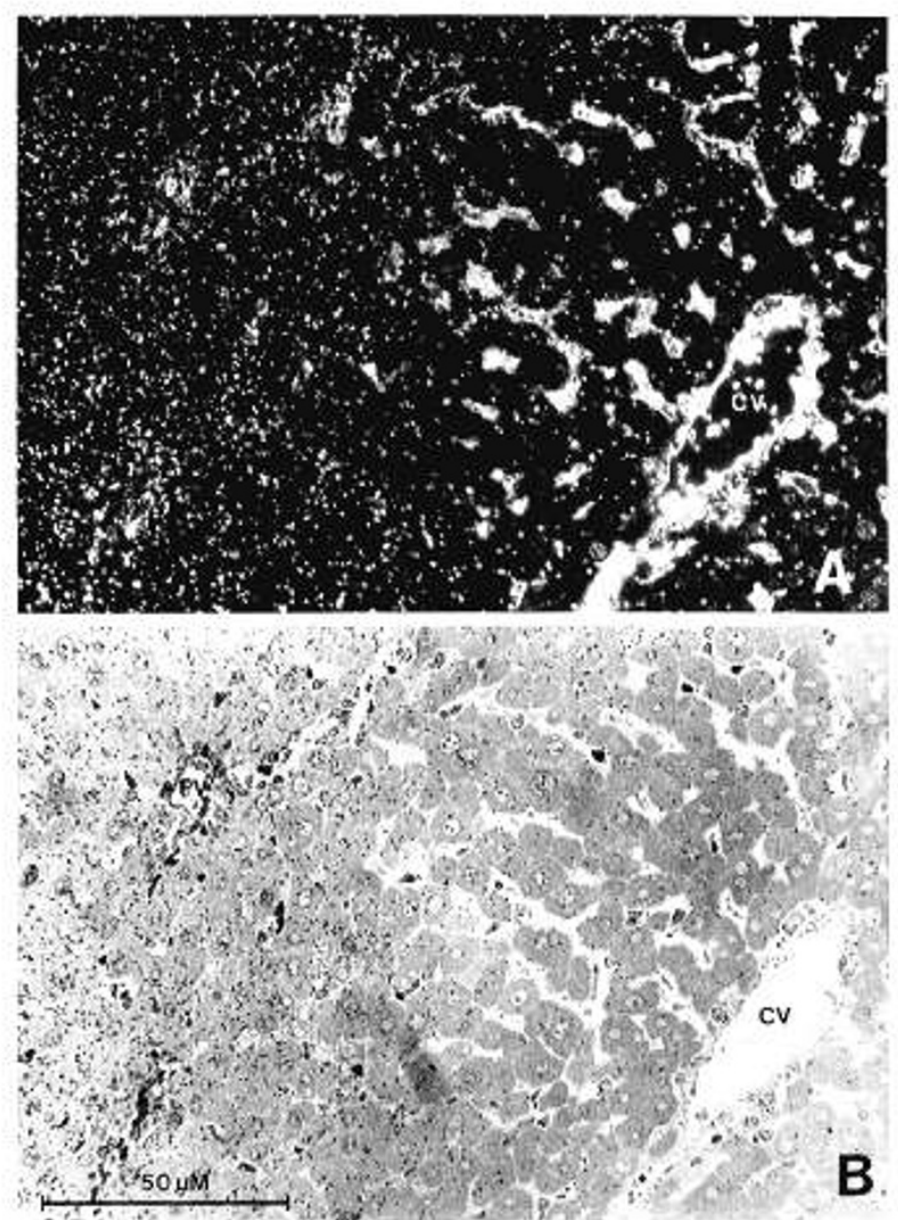

Fig. 1. Light microscope autoradiography of adult rat liver $30 \mathrm{~s}$ after portal vein injection of $\left[{ }^{125} \mathrm{I}\right]-\mathrm{CGT}$ using dark field $(A)$ and bright field $(B)$ microscopy. Silver grains in $A$ are presented as small white points. $P$, portal vein; $C V$, central vein.

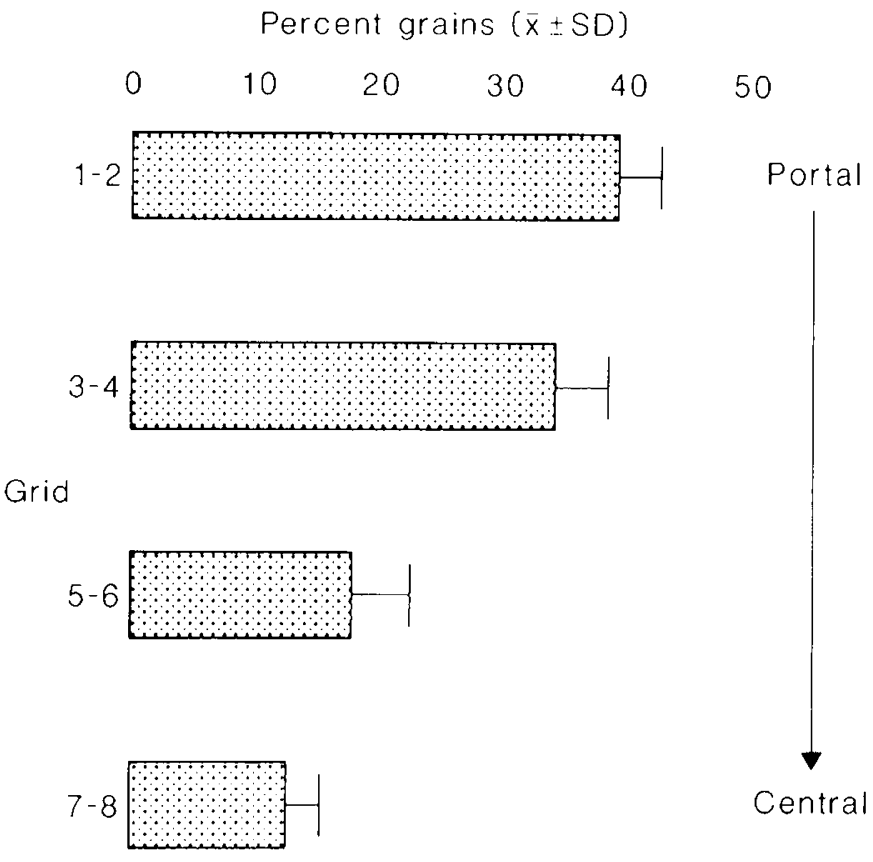

Fig. 2. Histograms showing the percent of total grains within each quarter of the hepatic lobule of the adult rat. Each bar represents the mean \pm SD of three separate experiments.

Serum bile acids were identified and quantified by combined capillary column gas chromatography-mass spectrometry after their isolation by liquid-solid and liquid-gel chromatography. These techniques have been described in detail elsewhere (14).

Statistical analysis. Serum bile acid concentrations in adult and suckling rats were compared using Student's $t$ test.

\section{RESULTS}

The autoradiographic demonstration of $\left[{ }^{125} \mathrm{I}\right] / \mathrm{CGT}$ distribution within the hepatic acinus of the adult rat $30 \mathrm{~s}$ after injection of tracer is shown in Figure $1 A$ using dark field illumination. The same field (Fig. $1 B$ ) shows the lobular detail under bright field illumination. There was a steep, decreasing portal (zone 1 of the acinus) to centrilobular (zone 3 ) gradient for the uptake of CGT. Approximately $40 \%$ of grains fell within the quarter of the lobule adjacent to the portal triad (Fig. 2). In contrast, the quarter segment closest to the central vein contained only $10 \%$ of the total number of silver grains.

Figure 3 illustrates the acinar distribution of CGT in the liver of the 14-day-old rat. There was no lobular gradient for the uptake of the isotope. The entire lobule appeared to be uniformly labeled; grain quantitation (Fig. 4) showed that approximately $25 \%$ of the grains were located within each quarter of the lobule.

Total bile acid concentrations were measured by combined gas chromatography-mass spectrometry in peripheral (systemic) and portal blood of 14-day-old and adult rats (Table 1). The portal vein bile acid concentration was significantly higher in the adult compared with the 14-day-old rats (total $86.4 \pm 25.4$ versus $35.4 \pm 2.1 \mu \mathrm{mol} /$ liter, $P \leqslant 0.01)$. Despite lower bile acid levels in the portal circulation, the total bile acid concentration was 10 -fold higher in the peripheral serum of developing rats $(229.4$ \pm 138.8 versus $20.3 \pm 2.3 \mu \mathrm{mol} /$ liter, $p \leqslant 0.02$ ). The higher peripheral to portal bile acid concentration ratio (6.48 at 14 days versus 0.23 in the adult) emphasizes the lower capacity of the developing liver to extract bile acids from portal blood.

Analysis of serum by combined gas chromatography-mass spectrometry revealed that cholic acid and its conjugates were the predominant bile acids in both peripheral and portal blood of the adult and 2-wk-old rat (Table 2). In contrast to the human, 
significant quantities of murocholic acid isomers (approximately $20-40 \%$ of the total) were detected in serum at each age.

\section{DISCUSSION}

The smallest microcirculatory unit of the liver, the acinus, is the site of solute exchange between portal blood and the hepatocytes (15). The liver acinus should be considered a threedimensional structure with blood flowing radially from the smallest (terminal) branches of the portal vein and hepatic artery into the sinusoids before draining into the terminal hepatic venules (15). Flow is unidirectional from an arbitrarily defined zone 1 nearest the portal structures, though an intermediate zone 2 , and finally to zone 3 , the periphery of the microvascular unit nearest the terminal hepatic venule (15). A gradient for solute uptake (portal to central) within the acinus could result from liver cell heterogeneity within the acinus, e.g. a greater number of solute carriers on the sinusoidal membrane of the hepatocytes in zone 1 compared to zone 3 . Alternatively, such a gradient may merely reflect a progressive decrease in the concentration of solute and thus a decrease in the amount available for uptake as blood flows sequentially through the sinusoids. Recent morphometric studies indicate that the surface to volume ratio of zone 1 sinusoids is significantly larger than that of zone 3 (16). This sinusoidal geometry would thus favor greater solute-plasma membrane interaction and possibly enhanced uptake in zone 1 of the liver acinus.

Evidence for an acinar gradient for bile acid transport in adult rat liver has been suggested by studies in which bile acid clearance and bile secretion were measured following damage to zone 1 or

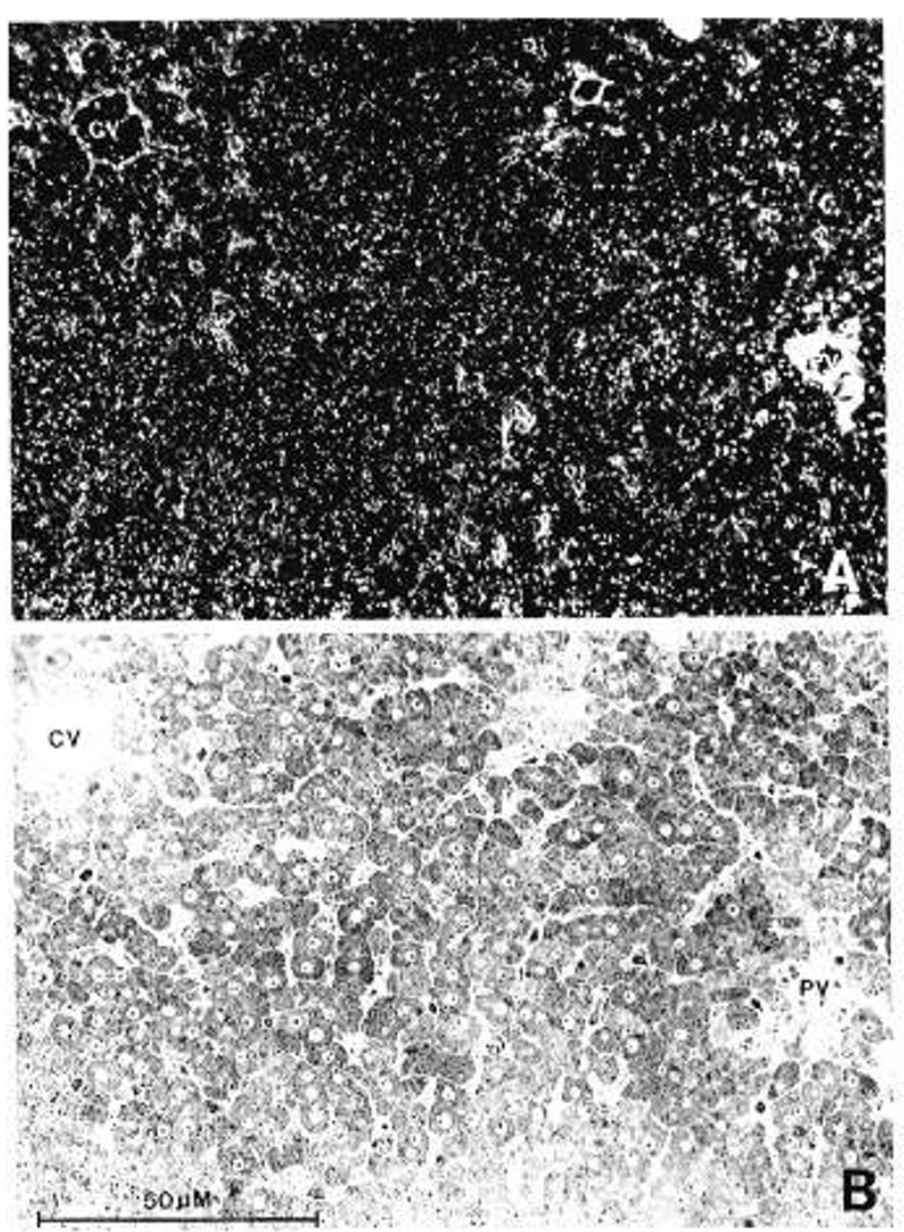

Fig. 3. Light microscope autoradiography of suckling rat liver $30 \mathrm{~s}$ after portal vein injection of $\left[{ }^{125} \mathrm{I}\right]-\mathrm{CGT}$ using dark field $(A)$ and bright field $(B)$ microscopy.

$$
\text { Percent Grains }(\bar{x}+S D)
$$

0

10

20

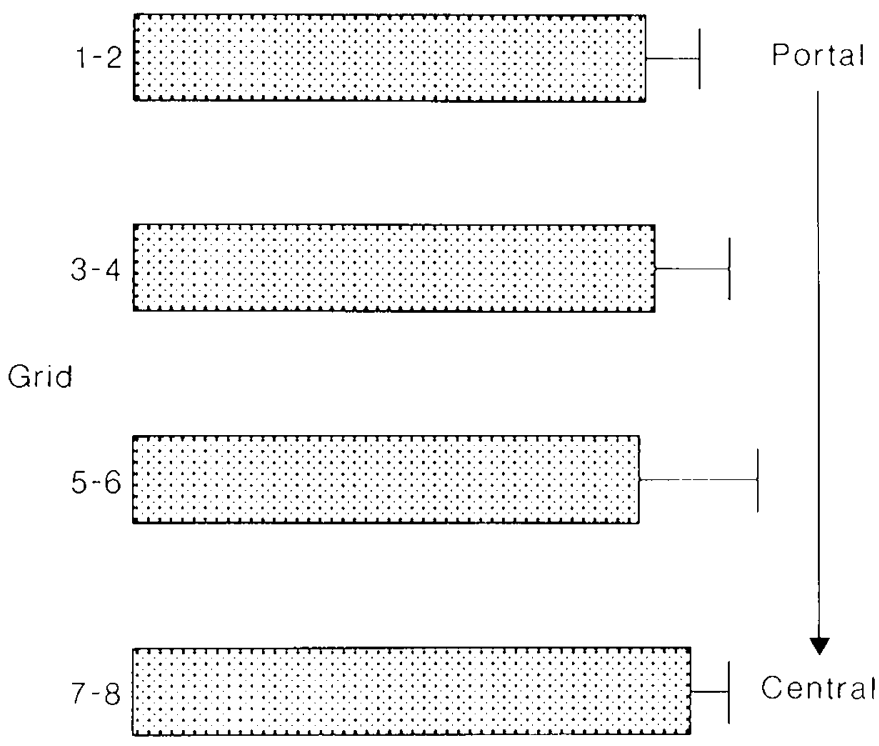

Fig. 4. Histograms showing the percent of total grains within each quarter of the hepatic lobule of the suckling rat. Each bar represents the mean \pm SD of three separate experiments.

zone 3 hepatocytes by selective toxins (17). In later studies a distinct portal to centrilobular gradient for bile acid uptake was demonstrated using autoradiographic techniques $(3,4)$. It remains uncertain whether intrinsic differences in the capacity for bile acid uptake exist among hepatocytes in the lobule. However, it is clear that all hepatocytes are potentially capable of bile acid transport as indicated by progressive recruitment of more of the acinus, including pericentral hepatocytes, with presentation of an increased bile acid load $(4,18)$. Autoradiographic localization of a bile acid tracer following retrograde perfusion of the label has shown that it is possible to actually reverse the lobular gradient to a decreasing zone 3 to zone 1 pattern (4). These data suggest that the acinar gradient for bile acid uptake is related more to the high clearance capacity of adult hepatocytes for bile acids rather than actual heterogeneity in the capacity of zone 1 and zone 3 cells for bile acid uptake (19).

We were unable to demonstrate an acinar gradient for bile acid uptake suckling rat liver. All acinar zones were uniformly labeled with silver grains. The pattern was similar to that observed in adult rat liver with progressive recruitment of cells within the acinus during bile acid infusion (4). It is likely that the entire hepatic lobule, including zone 3 hepatocytes, which normally serve in a "reserve" capacity, participates in the uptake of bile acids during development even under the basal conditions of this study. The markedly higher ratio of systemic to portal serum bile acid concentrations in the 14-day-old rat suggest that recruitment of these hepatocytes was not sufficient to compensate for decreased uptake resulting in high systemic concentrations of bile acids.

The mechanisms underlying the lack of an acinar gradient for bile acid uptake in developing rat liver remain undefined. However, elevated bile acid concentrations in peripheral serum $(6,7)$ and studies in hepatocytes (9) and plasma membrane vesicles (10) indicate that a decreased capacity for bile acid uptake may be the most important contributing factor. These transport studies showed that the $V_{\max }$ for taurocholate uptake in the 14-dayold rat was only $30-40 \%$ of the adult rate. The low taurocholate $\mathrm{V}_{\max }$ may reflect a decreased number of functional bile acid carriers on the basolateral plasma membrane during development. Ontogenic changes and the driving forces for the transport 
Table 1. Bile acid concentrations ( $\mu$ mol/liter) in mature and developing rat measured by gas chromatography and mass spectrometry

\begin{tabular}{|c|c|c|c|c|c|}
\hline \multirow[b]{2}{*}{ Bile acid } & \multirow[b]{2}{*}{ Structure } & \multicolumn{2}{|c|}{ Adult } & \multicolumn{2}{|c|}{2 wk old } \\
\hline & & $\begin{array}{l}\text { Portal vein } \\
\qquad(n=5)\end{array}$ & $\begin{array}{l}\text { Peripheral } \\
\quad(n=4)\end{array}$ & $\begin{array}{l}\text { Portal vein } \\
\quad(n=2)\end{array}$ & $\begin{array}{l}\text { Peripheral } \\
\quad(n=3)\end{array}$ \\
\hline Cholic & $3 \alpha, 7 \alpha, 12 \alpha-5 \beta$ & $36.8 \pm 7.1$ & $5.2 \pm 0.7$ & $8.8 \pm 6.2$ & $138.8 \pm 100.1$ \\
\hline Allo-cholic & $3 \alpha, 7 \alpha, 12 \alpha-5 \alpha$ & Trace & ND* & ND & $18.9 \pm 13.8$ \\
\hline Iso-cholic & $3 \beta, 7 \alpha, 12 \alpha-5 \beta$ & $6.1 \pm 4.8$ & $1.0 \pm 0.3$ & Trace-5.31 & $12.4 \pm 4.7$ \\
\hline Ursocholic & $3 \alpha, 7 \beta, 12 \alpha-5 \beta$ & $1.4 \pm 0.5$ & Trace- 2.2 & Trace & $4.1 \pm 1.7$ \\
\hline Chenodeoxycholic & $3 \alpha, 7 \alpha-5 \beta$ & $1.6 \pm 0.3$ & Trace-2.1 & ND & $5.4 \pm 4.0$ \\
\hline Deoxycholic & $3 \alpha, 12 \alpha-5 \beta$ & $6.3 \pm 2.8$ & $1.52 \pm 0.5$ & $5.1 \pm 0.4$ & $19.0 \pm 13.9$ \\
\hline$\alpha$-Muricholic & $3 \alpha, 6 \beta, 7 \alpha-5 \beta$ & $4.9 \pm 3.3$ & $1.85 \pm 0.8$ & Trace 2.61 & $15.7 \pm 21.7$ \\
\hline$\beta$-Muricholic & $3 \alpha, 6 \beta, 7 \beta-5 \beta$ & $11.5 \pm 4.6$ & $4.7 \pm 1.4$ & $10.04 \pm 1.1$ & $4.9 \pm 2.8$ \\
\hline$\omega$-Muricholic & $3 \alpha, 6 \alpha, 7 \beta-5 \beta$ & $2.3 \pm 1.3$ & $1.0 \pm 0.4$ & $4.07 \pm 0.06$ & $18.8 \pm 1.5$ \\
\hline Other muricholic isomers & & $7.7 \pm 2.1$ & $2.7 \pm 0.9$ & ND & $7.9 \pm 2.3$ \\
\hline Hyocholic & $3 \alpha, 6 \alpha, 7 \alpha-5 \beta$ & $4.9 \pm 1.8$ & $3.7 \pm 1.7$ & ND & $2.4 \pm 0.9$ \\
\hline Total & & $86.4 \pm 25.4$ & $20.3 \pm 2.3$ & $35.4 \pm 2.1$ & $229.4 \pm 138.8$ \\
\hline
\end{tabular}

* Not detected.

Table 2. Percent composition of bile acids in mature and developing rat measured by gas chromatography and mass spectrometry

\begin{tabular}{|c|c|c|c|c|c|}
\hline \multirow[b]{2}{*}{ Bile acid } & \multirow[b]{2}{*}{ Structure } & \multicolumn{2}{|c|}{ Adult } & \multicolumn{2}{|c|}{2 wk old } \\
\hline & & $\begin{array}{l}\text { Portal vein } \\
(n=5)\end{array}$ & $\begin{array}{l}\text { Peripheral } \\
(n=4)\end{array}$ & $\begin{array}{l}\text { Portal vein } \\
(n=2)\end{array}$ & $\begin{array}{l}\text { Peripheral } \\
\quad(n=3)\end{array}$ \\
\hline Cholic & $3 \alpha, 7 \alpha, 12 \alpha-5 \beta$ & $42.6 \pm 6.12$ & $25.34 \pm 2.5$ & $24.8 \pm 3.5$ & $55.3 \pm 17.85$ \\
\hline Allo-cholic & $3 \alpha, 7 \alpha, 12 \alpha-5 \alpha$ & Trace & ND* & ND & \\
\hline Iso-cholic & $3 \beta, 7 \alpha, 12 \alpha-5 \beta$ & $7.1 \pm 3.7$ & $5.2 \pm 1.8$ & Trace -15.0 & $7.16 \pm 4.5$ \\
\hline Ursocholic & $3 \alpha, 7 \beta, 12 \alpha-5 \beta$ & $1.6 \pm 0.4$ & Trace- 10.8 & Trace & $2.5 \pm 1.3$ \\
\hline Chenodeoxycholic & $3 \alpha, 7 \alpha-5 \beta$ & $1.9 \pm 0.25$ & Trace-10.4 & ND & $2.5 \pm 1.5$ \\
\hline Deoxycholic & $3 \alpha, 12 \alpha-5 \beta$ & $7.3 \pm 2.8$ & $5.6 \pm 1.7$ & $14.4 \pm 0.87$ & $5.4 \pm 3.0$ \\
\hline$\alpha$-Muricholic & $3 \alpha, 6 \beta, 7 \alpha-5 \beta$ & $5.7 \pm 3.2$ & $5.06 \pm 2.0$ & Trace- -7.4 & $7.23 \pm 4.1$ \\
\hline$\beta$-Muricholic & $3 \alpha, 6 \beta, 7 \beta-5 \beta$ & $13.3 \pm 1.16$ & $23.1 \pm 6.7$ & $28.6 \pm 3.5$ & $9.3 \pm 6.69$ \\
\hline$\omega$-Muricholic & $3 \alpha, 6 \alpha, 7 \beta-5 \beta$ & $2.7 \pm 0.3$ & $4.81 \pm 1.6$ & $11.49 \pm 0.5$ & $5.9 \pm 4.7$ \\
\hline Other muricholic isomers & & $13.0 \pm 4.4$ & $13.5 \pm 2.5$ & ND & $4.6 \pm 2.56$ \\
\hline Hyocholic & $3 \alpha, 6 \alpha, 7 \alpha-5 \beta$ & $5.7 \pm 0.7$ & $18.1 \pm 4.9$ & ND & $2.5 \pm 0.95$ \\
\hline Total & & 100.9 & 100.67 & 101.69 & 99.89 \\
\hline
\end{tabular}

* Not detected.

of muricholate isomers, which constitute a major proportion of the serum bile acid concentration in both age groups, have not been defined but the significantly higher muricholate serum levels in developing compared with adult rats would suggest that hepatic uptake of these bile acids is also decreased during development. It is therefore unlikely that the low transport capacity for bile acids is sufficient to produce a high enough initial extraction of bile acids from sinusoidal blood to generate an acinar gradient. A recent study examining the acinar distribution of BSP uptake in adult rat liver would support this notion (20). Autoradiographic studies showed that $\left[{ }^{35} \mathrm{~S}\right]$-BSP was uniformly distributed in the liver acinus following either normal or retrograde infusion. The authors suggested that since the initial extraction of labeled BSP was only $35-50 \%$ of the injected dose, a steep concentration gradient for the uptake of this compound would not be expected (20).

In addition, to a decreased number of bile acid carriers, structural features of the developing liver could also influence sinusoidal uptake. Although the surface area of the sinusoidal space is smaller in neonatal rat liver, it comprises a similar percent (approximately 10\%) of the liver volume as in the adult rat (21). However, it is not known if zonal differences exist in the surface to volume ratio of sinusoids as has been demonstrated for adult rat liver (16). Liver cell plates are often several hepatocytes thick in developing liver; this might result in less of the hepatocyte surface being exposed to portal blood. The dynamics of the portal circulation in the suckling rat may also differ from the adult. Preliminary studies, which utilized an isolated perfused suckling rat liver model, suggest that an increased volume of distribution may contribute to low rates of bile acid uptake prior to weaning (22).

In summary, we were not able to detect an acinar gradient for bile acid uptake in suckling rat liver. We propose that the lack of such a gradient may be related to the decreased transport capacity of the developing liver for bile acids. Our observations stress once again the minimal reserve function of the immature liver for bile acid transport and bile formation which may in part explain the increased susceptibility to cholestasis during early life.

Acknowledgment. CGT was a gift from Dr. P. Miller, Diagnostic Division of Abbot Laboratories, N. Chicago, IL.

\section{REFERENCES}

1. Blitzer BL, Boyer JL 1982 Cellular mechanisms of bile formation. Gastroenterology 82:346-357

2. Angelin B, Bjorkhem I, Einarsson K, Ewerth S 1982 Hepatic uptake of bile acid in man. Fasting and postprandial concentrations of individual bile acids in port venous and systemic blood serum. J Clin Invest 70:724-731

3. Jones AL, Hradek GT, Rensten RH, Wong KY, Karlaganis G, Paumgartner G 1980 Autoradiographic evidence for hepatic lobular concentration gradient of bile acid derivative. Am J Physiol 238:G233-G237 
4. Groothuis GMM, Hardonk MJ, Keulemans KPT, Nieuwenhuis P, Meijer DKF 1982: Autoradiographic and kinetic demonstration of acinar heterogeneity of taurocholate transport. Am J Physiol 243:G455-G462

5. Lester R 1980 Physiologic cholestasis. Gastroenterology 78:864-865

6. Suchy FJ, Balistreri WF, Heubi JE, Searcy JE, Levin RS 1981 Physiologic cholestasis: elevation of the primary serum bile acids in normal infants. Gastroenterology 80:1037-1041

7. Belknap WM, Balistreri WF, Suchy FJ, Miller P 1981 Physiologic cholestasis II. Serum bile acids reflect the development of enterohepatic circulation in rats. Hepatology 1:613-616

8. Klassen CD 1975 Hepatic excretory function in the newborn rat. J Pharmacol Exp Ther 184:721 -728

9. Suchy FJ, Balistreri WF 1982 Uptake of taurocholate by hepatocytes isolated from devcloping rats. Pediatr Res 16:282-285

10. Suchy FJ, Courchene SM, Blitzer BL 1985 Taurocholate transport by basolateral liver plasma membrane vesicles isolated from developing rats. Am J Physiol 248:G648-G654

11. Suchy FJ, Balistreri WF, Hung J, Miller P, Garfield SA 1983 Intracellular bile acid transport of rat liver as visualized by electron microscope autoradiography using bile acid analogue. Am J Physiol 245:G681-G689

12. Karnovsky MJ 1965 A formaldehyde-glutaraldehyde fixative of high osmolarity for use in electron microscopy. J Cell Biol 27:137(abstr)

13. Luft HH 1961 Improvements in epoxy resin embedding methods. J Biophys Biochem Cytol 9:409-414
14. Setchell KDR, Matsui A 1983 Serum bile acid analysis. Clin Chem Act 127:117

15. Rappaport AM 1973 The microcirculatory hepatic unit. Microvasc Res 6:212228

16. Miller DL, Zanolli CS, Gumucio JJ 1979 Quantitative analysis of the rat liver. Gastroenterology 76:965-969

17. Gumucio JJ, Balabaud C, Miller DL, Demason LJ, Appelman HD, Stoecker TJ, Franzblau DR 1978 Bile secretion and liver cell heterogeneity in the rat. J Lab Clin Med 91:350-362

18. Layden TJ, Boyer JL 1979 Recruitment of central lobular hepatocytes for bile acid-dependent bile secretion. In: Paumgartner G, Strehl A, Gerwok W (eds) Biological Effects of Bile Acids. MTP, Lancaster, England, pp 3-9

19. Gumucio JJ 1983 Functional and anatomic heterogeneity in the liver acinus: impact on transport. Am J Physiol 244:G578-G582

20. Groothuis GMM, Keulemans KP, Hardonk MJ, Meijer DK 1983 Acinar heterogeneity in hepatic transport of dibromosulfophtholein and ouabain studied by autoradiography, normal and retrograde perfusions and computer stimulation. Biochem Pharmacol 32:3069-3078

21. Daimon T, David H, Azlinicki TV, Marx E 1982 Correlated ultrastructural and morphometric studies on the liver during perinatal development of rats. Exp Pathol 21:237-250

22. Sunaryo FP, Watkins JB, Ling S 1982 Neonatal hepatic function: change in vascular volume of distribution influences bile acid uptake. Gastroenterology $82: 1247$ (abstr) 